\title{
Rational Approach to the Spare Tools and Accessories Stocking Model Selection for the Flight Servicing Radio-Technical Facilities in the Context of the Relationships Commercialization with Military Industrial Complex Enterprises
}

\author{
Igor V. Khomenko and Oleg D. Gizatullov* \\ Military Training and Research Center of the Air Force \\ «Air Force Academy ft. Professor N.E. Zhukovsky and Y.A. Gagarin» \\ 54a Starykh Bol'shevikov Str., Voronezh, 394064, Russia
}

Received 26.10.2015, received in revised form 04.12.2015, accepted 13.02.2016

\begin{abstract}
The analysis of the approaches to the development of the general methodical apparatus for the spare tools and accessories amount substantiation and their stock-list formation for the advanced flight servicing radio-technical facilities during their exploitation is performed in the article. The possibility of the spare tools and accessories list optimization and its compiling for the required reliability indexes providing is substantiated basing on the logical-probability method appliance in the new application domain of the weaponry reliability indexes evaluation and estimation of the certain hardware component significance and its contribution to the flight servicing radiotechnical facilities construction and their functionality. The mathematical apparatus and the algorithm for the concerned problem solving are suggested.
\end{abstract}

Keywords: reliability, spare tools and accessories, optimization, cost minimization.

Citation: Khomenko I.V., Gizatullov O.D. Rational approach to the spare tools and accessories stocking model selection for the flight servicing radio-technical facilities in the context of the relationships commercialization with military industrial complex enterprises, J. Sib. Fed. Univ. Eng. technol., 2016, 9(3), 350-359. DOI: 10.17516/1999-494X-2016-9-3-350-359.

C) Siberian Federal University. All rights reserved

* Corresponding author E-mail address: 077leg@yandex.ru 


\title{
Рациональный подход к выбору модели
}

комплектования запасными инструментами

и принадлежностями радиотехнических средств

\section{обеспечения полетов}

\section{в условиях коммерциализации взаимоотношений \\ с предприятиями военно-промышленного комплекса}

\author{
И.В. Хоменко, О.Д. Гизатуллов \\ ВУНЦ ВВС «ВВА им. проф. Н.Е. Жуковского и Ю.А. Гагарина» \\ Россия, 371600, Воронеж, ул. Старых Большевиков, $54 a$
}

В статье проведен анализ подходов к разработке общего для перспективных образияов радиотехнических средств радиотехнического обеспечения полетов методического аппарата обоснования объёмов и порядка создания номенклатуры запасных элементов и принадлежностей, обеспечивающих требуемые показатели надежности на этапе его эксплуатации. На основе применения логико-вероятностного метода в новой предметной области по оценке надежности показателей вооружения, значимости и вклада отдельного элемента (составной части) в конструкцию и функционирование образца радиотехнических средств радиотехнического обеспечения полетов обоснована возможность формирования перечня и оптимизации состава комплекта запасных инструментов и принадлежностей для обеспечения требуемых показателей надежности. Предложен математический аппарат и алгоритм решения задачи.

Ключевые слова: надежность, запасные инструменты и принадлежности, оптимизация, минимизация стоимости.

Современные образцы радиотехнических средств обеспечения полетов (РТС РТО) с физикотехнологической точки зрения представляют собой сложные структуры. Принципиальные и функциональные схемы проектирования, конструкция и технология изготовления радиотехнических средств радиотехнического обеспечения полетов должны обеспечивать такое протекание процессов, при котором изменение параметров изделий в определенных условиях в течение требуемого времени эксплуатации не приводило бы ни к временной, ни к полной потере их работоспособности. Потеря работоспособности РТС РТО может наступить как результат нестабильности (обратимое изменение) либо деградации (необратимое изменение) параметров изделий из-за старения материалов и износа отдельных деталей конструкции. Для обеспечения восстановления неисправной РТС РТО создаётся система запасных элементов и принадлежностей (ЗИП), которая обеспечивает своевременное обеспечение ремонтных бригад и расчётов необходимыми для восстановления запасными элементами. Одним из критериев, характеризующих эффективность системы запасных элементов, является безотказность эксплуатации РТС РТО. С безотказностью косвенно связаны такие экономические показатели, как длительность простоев техники, размер расходов на поиск и устранение причин предотказовых и аварийных ситуаций и др. Кроме того, эффективность системы запасных элементов, а также её экономическая целесообразность зависят от грамотного распределения и управления существующими ресурсами. 
В соответствии с ГОСТ РВ 0015-705-2008 различают следующие комплекты ЗИП: одиночный комплект - ЗИП-О;

групповой комплект - ЗИП-Г;

ремонтный комплект - ЗИП-Р [1].

Для обеспечения эксплуатации РТС РТО промышленностью разрабатываются и поставляются одиночные комплекты ЗИП и групповые комплекты ЗИП образцов, а также ремонтный комплект ЗИП для обеспечения ремонта изделий военной техники в ремонтных органах или на предприятиях промышленности, а также для пополнения других комплектов ЗИП.

Одним из основных условий обеспечения эксплуатационной надежности вооружения и военной техники (BBT) и оборудования (в дальнейшем - изделий) является оптимальная комплектация ЗИП, необходимых для устранения отказов, технического обслуживания и ремонта. В настоящее время для оценки и расчета количественных характеристик запасов восстанавливаемых и невосстанавливаемых составных частей изделий в одиночных и групповых комплектах ЗИП используются аналитические методики, приведенные в действующих государственных стандартах Российской Федерации. Методики основаны на математическом аппарате теории массового обслуживания и, в частности, марковских цепях с конечным числом состояний и с непрерывным временем, возможности которого существенно ограничивают круг решаемых задач. Подобные методики применяют для расчета параметров надежности отдельных радиоэлементов и для стран блока НАТО в соответствии с американским военным стандартом MIL-HDBK-217F, который предназначен для использования в качестве инструмента при оценке пригодности новых проектов и сравнения различных проектов, основан на использовании экспоненциального закона распределения.

Отечественными и зарубежными специалистами отмечается, что соответствующими стандартами не обеспечиваются показатели надежности с гарантированной точностью вследствие того, что законы распределения потоков отказов некоторых образцов техники (механические, электромеханические узлы и блоки, вычислительные устройства, группы элементов с резервированием и др.) значительно отличаются от экспоненциального закона распределения.

Используемые в настоящее время в промышленности методики расчета надежности восстанавливаемых систем при наличии ЗИП являются приближенными, имеющими знакопеременную погрешность, значение которой неизвестно как по знаку, так и по абсолютной величине. Выборочная проверка точности показывает, что существуют области, где погрешность достигает сотен и тысяч процентов: системы с групповым дублированием, дублированные системы при оценке показателей готовности [2].

Цена ошибки в расчетах стоимости комплектов ЗИП для указанных образцов существенно возрастает, поскольку стоимость комплектов ЗИП для них в отдельных случаях может быть сопоставима со стоимостью самого образца.

В большинстве современных работ по повышению эффективности методики комплектования ЗИП рассматриваются различные модели надёжности технических систем с применением методов динамического программирования, анализа и отсева вариантов для решения оптимизационной задачи. Однако использование таких подходов не позволяет распределить имеющиеся ресурсы для обеспечения основных эксплуатационно-технических характеристик РТС РТО - безотказности, ремонтопригодности, долговечности - и, следовательно, определить 
оптимальный вариант системы ЗИП. В современных условиях развития рыночной экономики широкое распространение получает система интегрированной логистической поддержки (ИЛП) выпускаемых образцов ВВТ на протяжении всего их жизненного цикла, что требует совершенствования (а в ряде случаев и пересмотра) методологической базы технического обеспечения, в том числе методического аппарата обоснования номенклатуры и достаточности ЗИП.

Система управления запасами составных частей РТС РТО, входящих в ЗИП различных типов, включает в свой состав:

органы военного управления, осуществляющие функции формирования потребности и анализа обеспеченности запасов ЗИП, планирования их заказов и распределения по системе складов и непосредственно образцам РТС РТО;

систему различных складов, осуществляющих функции накопления, хранения и распределения (и др.) запасов ЗИП.

Система управления запасами построена по иерархическому принципу: центр - объединения (соединения) - воинские части (подразделения) - образцы РТС РТО.

Для удовлетворения требований в поставках материальных средств комплектов ЗИП в центре на основе поступивших заявок осуществляется организация снабжения войск элементами требуемой номенклатуры, обобщение заявок от командований, определение потребности в элементах требуемой номенклатуры, планирование их заказов и поставок на основе выделенных бюджетных лимитов и возможностей предприятий-поставщиков (изготовителей) путём заключения в установленном порядке соответствующих государственных контрактов. Удовлетворение требований в поставках материальных средств комплектов ЗИП на предприятияхразработчиках изделий производится в три этапа:

определение исходных данных для расчета ЗИП, согласование их с заказчиком путем составления протокола согласования исходных данных для расчета ЗИП (если требования к комплектации ЗИП не заданы в техническом задании);

расчет одиночного и группового комплектов ЗИП;

составление ведомостей ЗИП и их согласование с заказчиком.

На каждом уровне структуры ЗИП могут быть использованы различные стратегии пополнения:

периодическое пополнение;

периодическое пополнение с экстренными доставками;

непрерывное пополнение;

пополнение по уровню $[2,3]$.

Базовым понятием для надежности комплекта ЗИП является «отказ комплекта ЗИП». Отказом комплекта ЗИП называется такое событие в системе «изделие - ЗИП», которое вызывает полную или частичную потерю работоспособности изделия при отсутствии в комплекте ЗИП соответствующей запасной части (ЗЧ). Из этого определения следует, что событие, называемое «отказ комплекта ЗИП», на самом деле связано не только с состоянием запасов, но и с состоянием системы, наличием или отсутствием структурного резерва [2].

Очевидно, что выбор показателей надежности решающим образом зависит от того, какие требования наиболее приоритетны. Полагается, что задаваемые (нормируемые) показатели надежности вместе с методами их проверки должны обеспечить уровень надежности, достаточ- 
ный для выполнения изделием его задачи. Эффективность зависит от надежности элементов, входящих в систему (факторы надежности обозначаются обычно множеством траекторий системы $G$ ), и других ненадежностных факторов (обозначаются символом множества $\Omega$, которому они принадлежат), тогда

$$
E=E(G, \Omega)
$$

где $E$ - эффективность.

Среднее значение реальной эффективности сложной системы определяется соотношениeм

$$
E=\sum_{m=0}^{n}\left\{\sum_{k=1}^{C_{n}^{m}} H_{m}^{(k)} E_{m}^{(k)}\right\}
$$

где $H_{m}^{(k)}$ - вероятность $S_{m}^{(k)}$ состояния системы, когда неработоспособны $m$ комплектующих элементов; $E_{m}^{(k)}-$ показатель эффективности $S_{m}^{(k)}$ состояния системы; $C_{n}^{m}=\frac{n !}{m !(n-m) !}-$ количество сочетаний; $n$ - общее количество элементов в системе; $m$ - количество неработоспособных элементов.

Число слагаемых в выражении расчета реальной эффективности равно числу возможных состояний и определяется соотношением

$$
\sum_{m=0}^{n} C_{n}^{m}=2^{n}
$$

C учетом приведенных выражений рассматриваемый показатель надежности применительно к образцу вооружения принимает вид уравнения

$$
K_{\ni \phi}=\sum_{m=0}^{n}\left\{\sum_{k=1}^{C_{n}^{m}} H_{m}^{(k)} \alpha_{m}^{(k)}\right\}
$$

где $\alpha_{m}^{(k)}=\frac{E_{m}^{(k)}}{E_{o}}-$ показатель относительной эффективности $S_{m}^{(k)}$ состояния образца РТС РТО, когда неработоспособны $m$ элементов.

Образцы РТС РТО относятся к многоцелевым изделиям конкретного назначения, которые состоят в общем случае из взаимосвязанных элементов различных номенклатур и предназначены для решения специальных задач. И процесс задания требований по надежности, предъявляемых к составным частям образца вооружения, представляет собой процесс оптимального синтеза сложной системы. Единственным практически реальным путем для проектирования и исследования таких систем является моделирование структурной надежности системы при использовании аппарата алгебры логики для описания процессов функционирования сложных систем. Однако именно для структурно-сложных систем характерной чертой выступает возможность и весьма сложных (многократных) комбинаций отказов элементов, каждая из которых невероятно мала, а в сумме таких «невероятных» событий 
набирается достаточно, чтобы система попала в состояние функционального отказа. Для постановки и решения задач моделирования и расчета надежности таких систем применим логико-вероятностный метод (ОЛВМ), в основе которого лежит так называемый событийнологический подход. Исходными данными для логико-вероятностных моделей надежности являются вероятности истинности отдельных аргументов в функции алгебры логики (ФАЛ), описывающих работоспособность системы; при этом возникает проблема статистической обработки малого числа наблюдений. Следует выделить два способа опытной оценки количественных характеристик показателей надежности:

по результатам специальных испытаний на надежность;

по результатам работы элементов в реальных условиях эксплуатации.

Однако первый способ связан с большими затратами, длительностью испытаний и даже с невозможностью их проведения. Второй способ получения значений показателей надежности не зависит от наблюдателя, который должен извлечь объективную информацию о надежности по данным разнородных источников.

Математическая модель работоспособности системы в виде логической функции работоспособности системы (ФРС) и полученная на ее основе вероятностная функция (5) позволяет оценить степень участия каждого из комплектующих элементов в общей характеристике всего образца РТС РТО.

$$
R_{c}=P\left\{y\left(x_{1}, x_{2}, . . x_{m}\right)=1\right\}, Q_{c}=P\left\{y^{\prime}\left(x_{1}^{\prime}, x_{2}^{\prime}, \ldots x_{m}^{\prime}\right)=1\right\},
$$

где $R_{c}$ - вероятность безотказной работы системы; $Q_{c}$ - вероятность отказа системы.

Понятие важности элемента предполагает знание влияния надежности элемента на общую надежность системы при выходе из строя того или иного элемента. Данная системная характеристика может быть оценена многими известными методами. В условиях отсутствия исходных данных о вероятностях отказов элементов целесообразно использовать один из двух методов определения значимости элементов системы - формально-топологический и функционально-топологический.

Первый метод основан на исследовании графа системы. Матрица непосредственных путей является основой для определения структурной значимости элемента системы - величины его ранга $r_{i}$, который отражает количество связей данного элемента с другими элементами и рассчитывается следующим образом:

$$
r_{i}=\frac{A_{i}+A_{i}^{2}}{\sum_{i=1}^{m}\left(A_{i}+A_{i}^{2}\right)},
$$

где $A_{i}$ - сумма строк матрицы непосредственных путей, имеющей единицы в главной диагонали.

Второй метод основан на анализе структурной схемы системы и описания принципа ее работы. На основании изучения функционирования системы строится граф, отражающий возможное доминирование элементов в системе, и матрица доминирования. Величину функционально-структурного ранга элемента предлагается вычислять по формуле (7).

$$
-355-
$$




$$
r_{i}=\frac{B_{i}+B_{i}^{2}}{\sum_{i=1}^{m}\left(B_{i}+B_{i}^{2}\right)},
$$

где $B_{i}$ - суммы строк матрицы смежности.

Обе оценки ранга, однако, не учитывают степени взаимных влияний элементов. Следует заметить, что другие методы оценки влияния отдельных элементов на надежность системы базируются на знании вероятностей исходных событий безотказной работы.

Так, надежностная значимость аргумента $x_{i}$ в системе с монотонной структурой определяется как частная производная

$$
B(i, R)=\frac{\partial R_{c}}{\partial R_{i}},
$$

где $R_{c}=f\left(R_{1}, \ldots, R_{m}\right)$ - вероятность безотказной работы системы.

Для учета неопределенности данных по значениям вероятностей безотказной работы значимость аргумента (элемента) трансформируется в структурную значимость, определяемую частной производной

$$
B(i)=\left.\frac{\partial R_{c}}{\partial R_{i}}\right|_{R_{i}=. .=R_{m}=0,5},
$$

представляющей значимость элемента при условии, что вероятности безотказной работы всех элементов равны 0,5 .

Сравнивая данное выражение значимости элемента с выражением

$$
\xi=\frac{\partial R_{c}}{\partial R_{i}}=P\left\{\Delta_{x_{i}} y\left(X_{m}\right)=1\right\} .
$$

и, подставляя вместо аргументов $x_{i}$ и $x_{i}^{\prime}$ константы значением 0,5 для всех $i=1,2, . . m$, выражение для веса важности элемента можно записать в виде:

$$
\left.g_{x_{i}}=P\left\{\Delta_{x_{i}} y\left(X_{m}\right)=1\right\}\right\}_{R_{1}=. . R_{m}=0,5} .
$$

Определение значимости элемента дает основное выражение для вычисления значимости элементов структурно-сложными системами (ССС), а значит, и образца РТС:

$$
\frac{\partial R_{c}}{\partial R_{i}}=R_{c 1}^{(i)}-R_{c 0}^{(i)}=\xi_{i}
$$

Таким образом, значимость элемента, означающая скорость изменения надежности всего образца РТС РТО, указывает на те комплектующие элементы, увеличение надежности которых на величину $\Delta R_{i}$ обеспечивает приращение надежности всего образца на величину

$$
\Delta R_{c}=x_{i} \Delta R_{i} .
$$

Значимость элемента определяется не только местом элемента в структуре образца вооружения, но и надежностью всех других элементов, кроме самого элемента $x_{i}$. Фактически значи-

$$
-356-
$$


мость элемента можно определить как условную вероятность безотказной работы образца при условии работоспособного состояния конкретного элемента.

Такой подход позволяет сформулировать понятие вклада элемента в надежность образца, который представляет собой произведение вероятности безотказной работы элемента $R_{i}$ на его значимость.

$$
B_{x_{i}}=R_{i} \frac{\partial R_{c}}{\partial R_{i}}=R_{i} \xi_{i}=R_{c}-R_{c 0}^{(i)}=R_{i}\left(R_{c 1}^{(i)}-R_{c 0}^{(i)}\right) .
$$

Из выражения (14) вытекает вывод о том, что вклад элемента есть та вероятностная добавка в безотказность образца РТС РТО, которую он приобретет при восстановлении отказа данного элемента и как приращение безотказности образца после введения данного элемента в структуру образца (в резерв).

В основу использования структурных схем для оценки значимости компонентов образца РТС РТО заложена концепция представления их в виде набора взаимодействующих компонентов, характеризуемых конечным набором свойств. При построении структурной схемы необходимо выявить и включить в рассмотрение те свойства реального образца РТС РТО, сочетание которых позволит с достаточной степенью определенности судить о том, насколько этот образец соответствует своему предназначению. Структурная модель строится на основе данных компонентной модели путем установления связей конструктивных компонентов образца РТС РТО друг с другом и, возможно, с элементами надсистемы (окружающей средой).

Принцип построения матрицы взаимосвязей функций и их носителей можно описать следующим образом. На горизонтальной оси размещаются данные о компонентах образца РТС РТО одного иерархического уровня описания структуры, а на вертикальной - главные функции этих компонентов, а также дополнительные, вредные и нейтральные функции рассматриваемой системы в целом. На пересечении колонок и строк отмечается участие каждого элемента в выполнении каждой функции. При этом для полезных функций в матрице фиксируется уровень их выполнения. Такой подход позволяет выявить неочевидные (скрытые) функции элементов объекта - как полезные, так и вредные. Через анализ матрицы по строкам устанавливается факт участия включенных в матрицу элементов объекта в выполнении конкретной функции.

Полученная форма перехода к полному замещению (ФППЗ) позволяет выполнить оценку значения вероятности безотказной работы всей рассматриваемой системы и, что важно, оценить значимость и вклад компонента, представленного в функциональной схеме. Как было указано ранее при изложении основ подхода к оценке величины влияния отдельных компонентов на безотказность систем образца РТС РТО, значимость компонента представляется частной производной логического дифференцирования вероятностной функции (ВФ) безотказной работы (13), а вклад - произведение значений значимости и вероятности безотказной работы (14).

Исходными данными для расчета являются: полином вероятностной функции рассматриваемого режима функционирования образца РТС РТО, числовые значения средней наработки до отказа всех компонентов (при рассмотрении их как невосстанавливаемых), требуемое время функционирования образца в модели данного сценария и режима.

$$
-357-
$$


Результатом является закон распределения времени безотказной работы образца РТС РТО по реализации функции, который предоставляет возможность расчета вероятности безотказной работы образца для заданной наработки.

При создании резерва запасных частей возникает задача не только обеспечения требований надежности, но и достижения заданных показателей надежности на этапе эксплуатации ограниченными средствами.

Постановка задачи по оптимизации состава придаваемых комплектов ЗИП для обеспечения заданных показателей надежности вооружения на этапе эксплуатации формулируется следующим образом: обеспечить максимально возможную вероятность безотказной работы образца РТС РТО на этапе эксплуатации при заданных затратах на резервные (запасные) составные части комплекта ЗИП-О.

Формализованная математическая запись формулировки задачи представляется следующими выражениями:

$$
\max \left(R_{c}\left(n, R_{i}, m_{i}, C\right)\right)
$$

при условии, что общая стоимость компонентов ЗИП-О

$$
C=\sum_{i=1}^{n}\left(C_{i} m_{i}+C_{o}^{i}\right) \leq C_{p}
$$

где $n$ - общее количество $i$ типов компонентов, для которых закладывается резерв; $m_{i}-$ количество резервных компонентов $i$ типа; $C_{i}$ - стоимость конструктивного компонента $i$ типа; $C_{o}^{i}$ - начальная стоимость резервированного компонента; $C_{p}$ - требуемая стоимость.

Задача оптимизации относится к области нелинейного или маргинального программирования, теория которого достаточно хорошо разработана и освещена.

Оптимизационный подход использует значения первых частных производных функции вероятности безотказной работы и в этом аналогичен методу градиентного спуска. Из приведенного выше следует, что, зная вероятность безотказной работы системы $R_{c}$ при некотором значении аргументов $\left\{R_{1}, R_{2}, \ldots, R_{i}, R_{n}\right\}$, можно легко вычислить значение $R_{c}^{\prime}$ при изменении одного аргумента и неизменных остальных $\left\{R_{1}, R_{2}, . ., R_{i}^{\prime}, . . R_{n}\right\}$ по следующей формуле:

$$
R_{c}^{\prime}=R_{c}+\frac{\partial R_{c}}{\partial R_{i}} \Delta R_{i}=R_{c}+\xi_{i} \Delta R_{i},
$$

где $R_{c}=\left\{R_{1}, R_{2}, \ldots, R_{i}, R_{n}\right\}$ - начальное значение показателя надежности системы; $R_{c}^{\prime}=R\left\{R_{1}, R_{2}, . ., R_{i}^{\prime}, . . R_{n}\right\}$ - значение показателя надежности системы при изменении значения вероятности безотказной работы компонента $i$.

Рассматривая положительный вклад компонента $i$ в общую надежность образца РТС РТО как долю значимости компонента, при изменении собственного показателя надежности от текущего до 1 можно утверждать, что, поскольку положительный вклад численно равен приращению надежности образца при увеличении надежности компонента, его значение можно использовать для выбора направления движения в алгоритме логико-вероятностных вкладов при возврате в область допустимых решений после нарушения ресурсных ограничений. 
При выборе ограничений стоимости компонентов ЗИП необходимо для каждого закладываемого в резерв элемента выбрать правило включения, в том числе, возможно, для каждого типа компонента $i$ индивидуально.

Перед началом работы алгоритма оптимизации необходимо подготовить следующие исходные данные:

построить логическую функцию работоспособности для образца РТС РТО (системы, подсистемы);

перейти от логической функции работоспособности к вероятностному полиному для расчета системного показателя надежности;

для всех возможных вариантов резерва состава компонентов (систем, подсистем) образца должна быть предварительно рассчитана их надежность и стоимость.

На первом шаге отыскивается такой компонент, прибавление к которому одного резервного элемента дает наибольший «удельный» выигрыш в приросте вероятности безотказной работы (коэффициенте готовности) в пересчете на одну единицу стоимости.

На втором шаге отыскивается следующий компонент, включая и пройденный, прибавление к которому резервного элемента дает наибольший прирост вероятности безотказной работы.

Таким образом, определив для каждого компонента рассматриваемого образца РТС РТО (его системы, подсистемы) на основе значимости реально достигнутого вклада и стоимости замены компонента, можно найти число и тип запасных компонентов, обеспечивающих максимально возможный показатель надежности в процессе эксплуатации по заданному сценарию.

\section{Список литературы}

[1] ГОСТ РВ 0015-705-2008 Запасные части, инструменты и принадлежности. Основные положения. М.: Стандартинформ, 2009. 18 с. [GOST RV 0015-705-2008 Spare parts, tools and accessories. The main provisions. M.: Standartinform, 2009. 18 (in Russian)]

[2] Черкесов Г.Н. Оценка надежности систем с учетом ЗИП: учеб. пособие. СПб.:БХВПетербург, 2012. 480 c. [Cherkesov G.N. // Evaluation of reliability of systems based on replacement elements and accessories: Textbooks. St. Petersburg: BHV-Petersburg, 2012. 480 (in Russian)]

[3] Тихонов В.И., Ефименко В.С., Журавлёв А.Г. Статистическая теория радиоэлектронных систем под редакцией В.И. Тихонова. Издание Военного авиационного технического университета, 2000. 691 c. [Tikhonov V.I., Efimenko V.S., Zhuravlev A.G. // Statistical theory of electronic systems, edited by V.I. Tikhonov. The publication of the Military Aviation Technical University, 2000. 691 (in Russian)]

[4] Петров А.И. Статистическая теория радиотехнических систем: учеб. пособие для вузов. М.: Радиотехника, 2003. 400c. [Petrov A.I. // Statistical theory of radio systems. Textbooks for higher education. AM: Radio, 2003. 400 (in Russian)]

[5] Математические методы военно-экономического анализа: учебник. Тверь, 2002. 422 с. [Mathematical methods of military-economic analysis. Textbook. Tver, 2002.422 (in Russian)] 